\title{
Essen und Soziale Arbeit
}

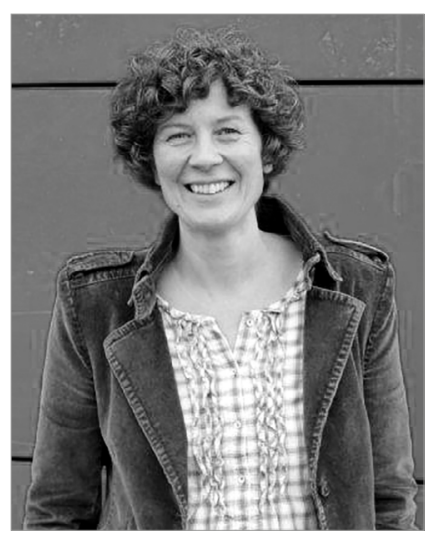

VON CHRISTINE MEYER

Prof. Dr. Christine Meyer ist Diplom-Sozialpädagogin und lehrt "Soziale Arbeit im Lebenslauf" an der Universität Vechta. Die Auseinandersetzung mit generationenübergreifender Arbeit und die Forschungen in Mehrgenerationenhäusern zeigte ihr, dass jüngere und ältere Menschen vor allem zu gemeinsamen Festen und zu gemeinsamen Mahlzeiten zusammentreffen. So kam das Thema "Essen« als Forschungsgegenstand in den Fokus ihrer Betrachtungen.

www.uni-vechta.de

\author{
Essen ist täglich ein Thema in fast allen sozialen \\ Einrichtungen, ohne dass dies oft konzeptionell \\ reflektiert wird. Soziale Arbeit erbringt auch bei \\ der Ernährung vielfache - fachlich weitgehend \\ unsichtbare - Hilfen, um eine selbstständige \\ Lebensführung zu unterstützen.
}

Die Essensversorgung als gesellschaftliche und soziale Herausforderung an die Freie Wohlfahrtspflege: Es geht nicht mehr nur ums Essen, aber immer auch!

Mehrmals am Tag nehmen Menschen Essen zu sich, denn sie benötigen die darin enthaltenen Nährstoffe für ihre tägliche Reproduktion und ihr Überleben. "Nahrung ist der Anfang von allem. Menschen müssen sich ernähren, und das Nahrungsbedürfnis haben Menschen vor allen anderen Nöten und Wünschen zu befriedigen.« (Barlösius 2011, S. 11)

Nahrung gehört zu dem ersten Bedürfnis von Menschen. Vom Tag der Geburt an werden Menschen von jemandem versorgt, überwiegend von der sie stillenden Mutter oder durch Flaschennahrung, die neben der Mutter auch vom Vater oder jeder anderen Personen gegeben werden kann. Mit dem Stillen und dem allmählichen Wechsel zu festerer Nahrung sind vielfältige Erziehungsprozesse verknüpft (vgl. Seichter 2012; 2014).

Menschen zählen zu den Omnivoren und können als solche lernen, insbesondere in der Kindheit, alles zu essen, was ihnen ihr Umfeld als genießbar anbietet (vgl. Schmidt 2011, S. 56). Damit eröffnet sich ihnen eine unendliche Fülle an zur Verfügung stehenden Nahrungsmitteln. Mit der Erziehung und Sozialisation werden Menschen auch über die tägliche Nahrung in die Ernährungsweisen und Esskultur der jeweiligen Gesellschaft eingeführt.

Essen ist für jeden Menschen lebensnotwendig und nicht für jeden gleich, wenn es um Selbstbestimmtheit, Selbstständigkeit und Unabhängigkeit geht,

\begin{tabular}{|l|c|c|}
\hline 1. Gesundheitshilfe & 7.481 & 192.005 \\
\hline 2. Jugendhilfe & 38.367 & 2.076 .693 \\
\hline 3. Familienhilfe & 4.570 & 41.082 \\
\hline 4. Altenhilfe & 18.051 & 520.727 \\
\hline $\begin{array}{l}\text { 5. Behindertenhilfe } \\
\text { 6. Hilfe für Personen in besonderen sozialen } \\
\text { Situationen }\end{array}$ & 16.446 & 509.395 \\
\hline 7. Weitere Hilfen & 8.830 & 53.650 \\
\hline $\begin{array}{l}\text { 8. Aus-, Fort- und Weiterbildungsstätten } \\
\text { für soziale und pflegerische Berufe }\end{array}$ & 9.914 & 242.447 \\
\hline Gesamt & 1.636 & 66.246 \\
\hline
\end{tabular}

(Quelle: BAG der Freien Wohlfahrtspflege 2014, S. 14)

Die Dienste und Einrichtungen der Freien Wohlfahrtspflege versorgen täglich mehrere Millionen Menschen. In diesen Angeboten muss oft auch eine Essensversorgung für die dort arbeitenden und versorgten Menschen stattfinden. 
wie beispielsweise in Bezug auf die Auswahl der Nahrungsmittel, die Vielfalt der Gerichte, die Entwicklung des Geschmacks, der Zubereitung sowie esskultureller Vorlieben oder gemeinsamer Mahlzeiten mit anderen Menschen.

\section{Essen und Freie Wohlfahrtspflege}

Unter dem Dach der Spitzenverbände der Freien Wohlfahrtspflege findet sich ein Großteil an Organisationen zusammen, wie Initiativen, Vereine, Stiftungen sowie soziale Dienste und Einrichtungen, die unterschiedliche Hilfen anbieten, um gesellschaftliche und soziale Herausforderungen abzufedern (vgl. Bundesarbeitsgemeinschaft (BAG) der Freien Wohlfahrtspflege 2014, S. 46).

Bundesweit wurden im Jahr 2012 in der Freien Wohlfahrtspflege 105.295 Einrichtungen und Dienste mit insgesamt 3.702.245 Betten oder Plätzen erfasst. In den Einrichtungen und Diensten sind 1.673.861 Mitarbeiterinnen und Mitarbeiter hauptamtlich beschäftigt. Darüber hinaus engagieren sich 2,5 bis 3 Millio-
Wenn Menschen aufgrund ihres Lebensalters oder ihrer Beeinträchtigungen auf die Hilfe anderer Menschen angewiesen sind und Unterstützung rund um das Essen benötigen, fühlen sie sich täglich in ihrer Existenz bedroht, wenn sie nicht ihren eigenen Vorstellungen von Essen und damit verbundenen Ernährungsweisen und Esskulturen folgen können. Nichts erscheint deshalb erst einmal so existenziell bedeutend, wie die Möglichkeit, eigene Vorstellungen zum Essen entwickeln zu können, vor allem auch über das eigene Ausprobieren und das Verwirklichenkönnen dieser Ideen.

\section{Essen in sozialen Diensten und Einrichtungen}

Für alle Menschen, die sich autonom versorgen können, erscheint beinahe nichts banaler als die tägliche Versorgung mit Nahrung. Für Menschen, die aus unterschiedlichen Gründen nicht selbsttätig, selbstbestimmt und unabhängig ihre eigene Nahrung auswählen, zubereiten und zu sich nehmen können, entstehen

\section{"Die Dienste und Einrichtungen der Freien Wohlfahrtspflege müssen täglich für mehrere Millionen Menschen - Nutzer wie Mitarbeitende - Mahlzeiten anbieten«}

nen freiwillig sozial, in den Hilfswerken, Initiativen und angeschlossenen Selbsthilfegruppen (vgl. ebd. 2014, S. 10).

In all den Organisationen werden täglich mehrere Millionen Menschen mit Essen oder verschiedenen Mahlzeiten versorgt. Ein Mensch kann nicht ohne Nahrung sein und mit dem Wissen um die existenzielle Bedeutung des Essens, wird das Maß der Abhängigkeit deutlich, wenn ein Mensch aufgrund von Beeinträchtigungen nicht in der Lage ist, für sein Essen selber sorgen zu können. Je nach Lebensalter, Grad der Beeinträchtigung und Lebensmittelpunkt haben die jeweiligen unterstützenden Fachkräfte und dazugehörige Institutionen, in denen Essen angeboten wird, erheblichen Einfluss auf die »Essbiographien $\ll$. diese existenziell bedrohlichen Situationen als Ausgeliefertsein dem anderen gegenüber bezüglich der eigenen Nahrungsauswahl und -zubereitung.

Alle Einrichtungen personenbezogener Dienstleistungen, die Essen anbieten, verfolgen mit ihren Mahlzeiten entweder konzeptionelle, vielleicht auch gleichzeitig erzieherische, sozialpädagogische, pflegerisch notwendige oder über Beeinträchtigungen beeinflusste Ideen beim Essen.

Die Freie Wohlfahrtspflege stellt in Deutschland in zahlreichen Handlungsfeldern das größte Angebot an sozialen Dienstleistungen bereit. Mit den Hilfeangeboten wird ein breit gefächertes Angebot qualifizierter sozialer Hilfen ermöglicht, zu denen vielfältige Angebote entlang aller Lebensalter und Lebenslagen gehören:
- »Angebote für Kinder und Jugendliche, Kindertagesstätten, Erziehungsberatung und Freizeitangebote, Hilfen für Familien und Alleinerziehende wie Ehe- und Schwangerschaftsberatung, Interventionsstellen, Familienpflege, Frauenhäuser

- Hilfen für alte Menschen wie Seniorentreffs, Mahlzeiten- und Besuchsdienste, vollstationäre Alten- und Pflegeheime und ambulante Pflegedienste sowie Seniorentagesstätten

- Dienste für Menschen mit Behinderung wie Frühförderung, Kindergärten und Schulen, Berufsförderungsund Berufsbildungswerke, Tagesstätten und Wohnheime

- Pflege von Kranken in Krankenhäusern,

- Tageskliniken, Tagespflegeeinrichtungen, Hilfe durch Vorsorge- und Rehabilitationskliniken und Beratungsstellen

- Angebote für Menschen mit Migrationshintergrund wie Ausländersozialberatung, Aussiedlerberatung, Psychosoziale Zentren für Flüchtlinge, Integrationsprojekte

- allgemeine Auskunfts- und Sozialberatungsstellen und ambulante Dienste, Nachbarschaftszentren, Suppenküchen und Mobile Soziale Dienste

- Hilfe für Menschen in sozialen Notlagen wie Obdachlosenunterkünfte, Schuldnerberatung, Bahnhofsmission, Telefonseelsorge

- Kontakt-, Informations- und Beratungsstellen für Selbsthilfegruppen und Gruppen bürgerschaftlichen Engagements (Freiwilligenzentren und Freiwilligenagenturen)

- Aus-, Fort- und Weiterbildungsstätten für soziale und pflegerische Berufe« (ebd. 2014, S. 7).

In allen genannten Einrichtungen finden entweder mehrmals täglich Essensangebote statt oder es wird einmalig beispielsweise in einem Beratungssetting etwas zum Verzehr im Rahmen der Gastlichkeit angeboten, wie Kaffee oder Tee. Darüber hinaus finden in Einrichtungen, je nach Zielsetzung, Projekte statt, die sich auch um gemeinsames Essen drehen, wie Suppenfeste, interkulturelle Frühstücke und gemeinsame Mittagstische. Essen ist täglich Thema in allen Einrichtungen. 


\section{Essen im gesellschaftlichen Kontext}

Essen und der Umgang mit Lebensmitteln sind abhängig von gesellschaftlichen Rahmenbedingungen und Regeln, Entwicklungen und historischen Ereignissen, die das Ernährungsverhalten prägen und bestimmen.

Die kulturell-historische Zeit, in die jemand hineingeboren wird und damit verbunden zeithistorische, technische und technologische Entwicklungen sowie politisch-wirtschaftliche Rahmenbedingungen und nicht zuletzt Lebensmittel und Zubereitungsarten, die in einem bestimmten historischen Zeitfenster auf
Altenpflegeheime, nachbarschaftliche Essensgemeinschaften, von sozialen Dienstleistern angebotene Mittagstische etc. Bereits 2012 weist die Freie Wohlfahrtspflege in diesen Bereichen Schwerpunktsetzungen auf (vgl. Tabelle).

Als größter Arbeitsbereich der Freien Wohlfahrtspflege hinsichtlich der Einrichtungszahl sowie der Bettenund Platzzahl gilt die Jugendhilfe mit 38.367 Einrichtungen und Diensten sowie 2.076.693 Betten und Plätzen. Die Alten- und Behindertenhilfe folgen mit 18.051 und 16.446 Einrichtungen und Diensten (vgl. ebd. 2014, S. 11).

Die Wohlfahrtsverbände nehmen in der Jugendhilfe innerhalb der freien Trä-

\section{"Viele Menschen nehmen mindestens eine Mahlzeit pro Tag außer Haus ein"}

dem Markt sind oder als "neu « auf den Markt kommen, haben (lebenslangen) Einfluss auf die Esskultur (vgl. Brombach et al. 2014, S. 12). Erlernte Muster aus der Kindheit in Bezug auf Ernährung und Esskultur werden über die Kindheit hinaus durch weitere soziale, gesellschaftliche, religiöse oder kulturelle Erfahrungen geprägt.

Bei Entscheidungen für das eine oder andere Nahrungsmittel wird von jeher eher auf den vertrauten Geschmack gesetzt oder das bereits bekannte Nahrungsmittel gewählt und weniger beispielsweise nach gesundheitlichen Aspekten entschieden. »Die frühen Erfahrungen eines Kleinkindes mit dem Essen prägen die Essgewohnheiten ein Leben lang. Erwachsene lernen immer noch weiter, das grundlegende Ess->Programm $<$ ist jedoch vorhanden. Es lässt sich nur durch bewusste Anstrengung ändern, wie die langjährigen Erfahrungen in der Ernährungsberatung zeigen. Essen wird also in der Kindheit sowohl durch als auch beim Essen gelernt." (Schmidt 2011, S. 55)

Für die Zukunft wird der außer Haus stattfindenden Essensversorgung hohes Wachstum vorhergesagt. Vor allem Kinder und Jugendliche sowie ältere Menschen werden diejenigen sein, die in Gemeinschaftsverpflegungen mindestens eine Mahlzeit pro Tag einnehmen werden. Dazu gehören vor allem Kindertagesstätten, Schulen, Horte, Bürgerzentren, Mehrgenerationenhäuser, ger eine bedeutende Rolle ein, da $80 \%$ aller durch freie Träger zur Verfügung stehenden Plätze im Bereich der Kindetagesbetreuung sowie knapp $58 \%$ der Plätze in den anderen Betreuungsfeldern der Jugendhilfe durch einen der Wohlfahrtsverbände bereitgestellt werden. Im Jahr 2013 wurden 1,6 Millionen Kinder innerhalb einer Einrichtung der Wohlfahrtsverbände betreut und 2012 befanden sich im Bereich der Gesundheitshilfe 36 Prozent der Krankenhäuser mit rund einem Drittel (34 Prozent) aller Betten und Plätze in den Händen der Freien Wohlfahrtspflege. Die Freie Wohlfahrtspflege stellt im Bereich der stationären Altenhilfe 2011 mehr als die Hälfte (54 $\%)$ aller Pflegeheime zur Verfügung (vgl. ebd. 2014, S. 12).

Damit steht die Freie Wohlfahrtspflege täglich mehrmals in der Verantwortung, für mehrere Millionen Menschen Mahlzeiten anzubieten. Dabei ist völlig ungewiss, welche Ideen die Essensversorgung begleiten, beispielsweise welche ernährungs- und gesundheitswissenschaftlichen, sozialpädagogischen oder heilpädagogischen Ideen mit den Mahlzeiten verfolgt werden.

Die Arbeiterwohlfahrt, der Deutsche Caritasverband, das Deutsche Rote Kreuz, der Paritätische Wohlfahrtsverband, die Diakonie Deutschland und die Zentralwohlfahrtsstelle der Juden in Deutschland haben ihre Wurzeln in unterschiedlichen Religionen und Welt- anschauungen, die ebenfalls mit bestimmten esskulturellen Ausprägungen, mit ethischen oder religiösen Regeln in Verbindung gebracht werden können und den Einrichtungen möglicherweise nach wie vor Orientierung in esskultureller Hinsicht bieten (vgl. ebd. 2014, S. 46).

Doch darüber ist bisher wenig bekannt. Seltsamerweise ist die Organisation und Durchführung des Essens scheinbar so alltäglich, da es so wenig Beachtung findet oder beforscht wird im Hinblick auf die damit verbundenen konzeptionellen Ideen. Obwohl fast alle genannten Hilfen historisch immer auch die Grundversorgung mit Nahrung darstellten (vgl. Meyer 2018).

\section{Essen und Lebensqualität}

Essen ist über die physische Notwendigkeit hinaus mit vielfältigen bedeutsamen Aspekten verknüpft, wie Identität, Zugehörigkeit, Kultur, Gemeinschaft, Selbstständigkeit, Unabhängigkeit, Genuss, Lebensrhythmus oder zunehmend bedeutend werdenden Aspekten, wie Gesundheit und Nachhaltigkeit (vgl. ebd. 2018).

Die Aufzählung könnte um viele weitere Perspektiven erweitert werden, die für Menschen wichtig sind, vor allem auch im Hinblick auf die Ausprägung ihrer eigenen Ernährungsbiographie. Selbstverständliche, bisher fachlich zu unsichtbare, Hilfen werden erbracht, um Menschen in einer unabhängigen Lebensführung zu unterstützen in Erziehung, Pflege oder Sozialem.

Hinter dem Oberbegriff »Essen « verbirgt sich dabei jeweilig eine komplexe Vielzahl an unterschiedlichen Tätigkeiten: Das Wissen um Lebensmittel und die damit verbundene Auswahl der Lebensmittel, der Einkauf, das Wissen um Rezepte, das Zubereiten und Kochen, die gemeinsame Mahlzeit als besondere Situation mit der dazugehörigen Tischund Esskultur inklusive Benimmregeln.

Die Entscheidung für Ernährungsweisen - vegan oder vegetarisch, bio oder herkömmlich, Außer-Haus-Verpflegung oder zu Hause - gehören dabei ebenso zu bedeutsamen essens- und damit lebensqualitätsbezogenen Entscheidungen, die jeden Tag mehrfach getroffen werden.

Essen ist dabei vor allem an den Orten von Bedeutung für die Freie Wohlfahrtspflege, wo Menschen wohnen, sich bilden, arbeiten oder betreut werden: in 


\section{Organisationen der Sozialwirtschaft im Umbruch}

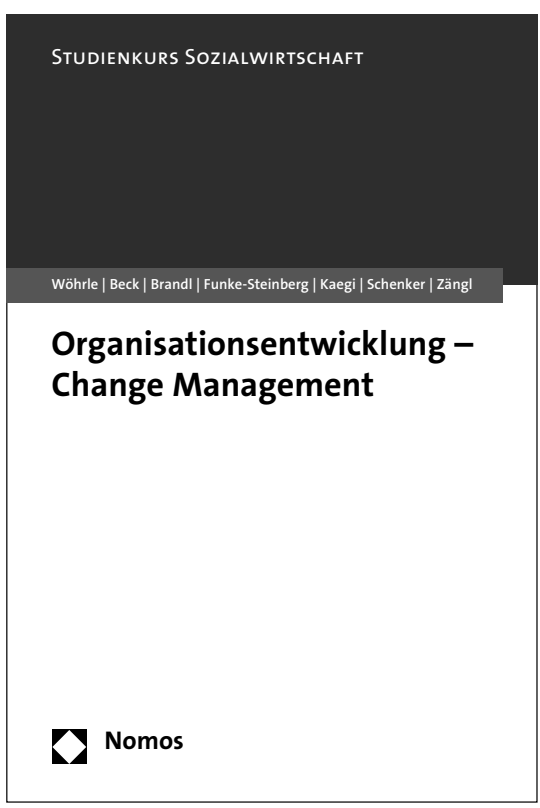

\section{Organisationsentwicklung - Change Management}

Von Prof. Dr. Armin Wöhrle, Prof. Dr. Reinhilde Beck,

Prof. Dr. Paul Brandl, Karsten Funke-Steinberg, Prof. Dr. Urs Kaegi,

Dominik Schenker und Prof. Dr. Peter Zängl

2019 , ca. 250 S., brosch., ca. 24,90€

ISBN 978-3-8487-4457-2

Erscheint ca. Februar 2019

nomos-shop.de/30502

Das führende Lehrbuch zum Management in der Sozialwirtschaft bietet eine systematische Einführung in die Grundlagen des Sozialmanagements und stellt Bezüge zur Sozialwirtschaft, Sozialpolitik, Sozialen Arbeit und den Wirtschaftswissenschaften her. In sechs Kapiteln erläutert das Lehrbuch die Begrifflichkeiten, behandelt die Geschichte des Sozialmanagements und gibt Einblicke in die sozialstaatlichen Schnittstellen sowie die gegenwärtigen Problemstellungen. Anhand eines Überblicks über aktuelle Diskussionspunkte werden die Besonderheiten und das Selbstverständnis des Fachs strukturiert herausgearbeitet.

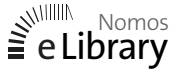

Bestellen Sie jetzt telefonisch unter (+49)7221/2104-37. Portofreie Buch-Bestellungen unter www.nomos-shop.de Alle Preise inkl. Mehrwertsteuer der eigenen Häuslichkeit, in ambulanten Settings, in Tageseinrichtungen, in teilstationären und stationären Einrichtungen. Je nach Alter, Beeinträchtigung oder Hilfe- oder Pflegebedürftigkeit einerseits und Zeit, Angebot, Kosten sowie Anspruch an Fachlichkeit, Gesundheit oder Nachhaltigkeit andererseits wird das Essen täglich mit dazugehörigen Mahlzeiten durchgeführt.

Die mit dem Essen verbundene Komplexität zieht den Anspruch nach sich, Fragen des Essens stärker in Aus-, Fortund Weiterbildung einzubinden sowie den vielfältigen Essenspraktiken mehr Sichtbarkeit in der Sozialen Arbeit als Lebensqualitätsgarant zu verschaffen.

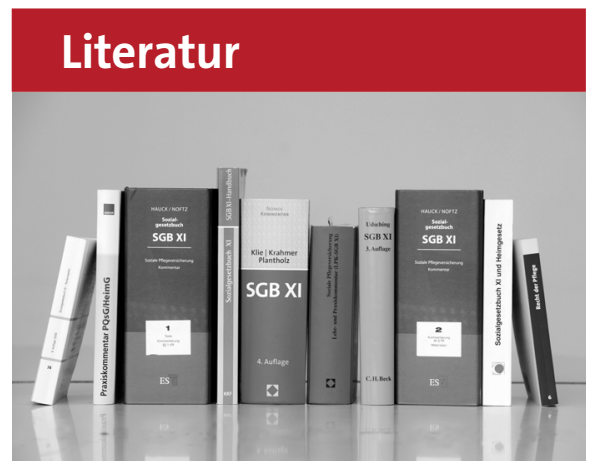

Barlösius, E. (2011): Soziologie des Essens. Eine sozial- und kulturwissenschaftliche Einführung in die Ernährungsforschung. Grundlagentexte Soziologie. 2., völlig überarbeitete und erweiterte Auflage. Weinheim: Beltz/Juventa.

Brombach, C./Haefeli, D./Bartsch, S./Winkler, G. (2014): Ernährungsmuster im Verlauf von drei Generationen: Gibt es inter- und intraindividuelle Unterschiede? In Internationaler Arbeitskreis für Kulturforschung des Essens. Mitteilungen 2014, S.H. 21: 12-22.

Bundesarbeitsgemeinschaft (BAG) der Freien Wohlfahrtspflege e. V. (Hg.) (2014): Einrichtungen und Dienste der Freien Wohlfahrtspflege. Gesamtstatistik der Freien Wohlfahrtspflege 2012. Berlin

Meyer, C. (2018): Essen und Soziale Arbeit. Eine Einführung. Wiesbaden: Springer VS.

Schmidt, S. (2011): Wie Kinder beim Essen essen lernen. In: Schönberger, G./Methfessel, B. (Hg.): Mahlzeiten. Alte Last oder neue Lust? Wiesbaden: VS Verlag, S. 55-71.

Seichter, S. (2014): Erziehung an der Mutterbrust. Eine kritische Kulturgeschichte des Stillens. Weinheim/Basel: Beltz Juventa. Seichter, S. (2012): Erziehung und Ernährung. Weinheim/Basel: BeltzJuventa. 